\title{
Commentary An anti-inflammatory role for tranexamic acid in cardiac surgery?
} Heidi J Robertshaw ${ }^{1,2}$

\author{
1St George's Hospital, Blackshaw Road, London SW17 0QT, UK \\ 2Department of Anaesthesia, Alice Springs Hospital, Gap Road, Alice Springs, NT 0870, Australia
}

Corresponding author: Heidi J Robertshaw, hroberts@sghms.ac.uk

Published: 16 January 2008

This article is online at http://ccforum.com/content/12/1/105

(c) 2008 BioMed Central Ltd

Critical Care 2008, 12:105 (doi:10.1186/cc6210)

See related research by Jimenez et al., http://ccforum.com/content/11/6/R117

\begin{abstract}
Pro- and anti-inflammatory cytokines are elevated after cardiac surgery. The control of the release of these major paracrine proteins is becoming clearer and they have been shown to be involved in the activation of the coagulation/fibrinolysis pathway, among other cascades. The association of a predominance of proinflammatory cytokines with morbidity in some patients, particularly following cardiac surgery, is well described but still incompletely understood. Clinical studies elucidating how clinicians may influence this cytokine release directly will improve our knowledge of the processes involved and could ultimately show benefit in better outcomes for patients.
\end{abstract}

Cytokines are an intriguing group of soluble protein mediators with a large number of described actions but short biological range. These molecules are produced by a variety of cells, including monocytes, macrophages, lymphocytes, and endothelial cells. Such proteins include the pro-inflammatory cytokines interleukin (IL)-6 and soluble tumour necrosis factor receptor-1 (sTNFR-1), as measured by Jimenez and colleagues [1] in their study. Cytokines have been shown to be important in all biological processes [2], including inflammation in which the major pro-inflammatory cytokines are considered to be tumour necrosis factor (TNF), IL-1, and interferon-gamma in addition to IL-6. They also have anti-inflammatory roles (IL-10, transforming growth factor-beta, and IL-4). The control of this balance between pro- and anti-inflammatory cytokines is vital to the understanding of inflammation and the inflammatory response in human disease processes.

Cytokines are normally subject to tight homeostatic control and are produced in response to a variety of physiologic and pathologic stimuli. Pro-inflammatory cytokines play a pivotal role in initiating and amplifying the inflammatory process. Furthermore, the plasma concentrations of certain cytokines, such as IL-1 and IL-6, have been shown to be predictive of outcome in specific subgroups of critically ill patients [3]. In particular, TNF and IL-1 are elevated early following cardiac surgery, with IL-6 and IL-8 peaking later $[3,4]$. This damaging pro-inflammatory cytokine response is well documented in patients who develop systemic inflammatory response syndrome (SIRS) after cardiac surgery [4]. In this SIRS patient group, IL-8 and IL-18 are higher in non-survivors compared with survivors. The association of elevated levels of pro-inflammatory cytokines with poor outcomes following cardiac surgery has been demonstrated by many studies [5], but a direct cause-and-effect relationship has not been demonstrated.

The pro-inflammatory cytokine response to injury, including cardiac surgery, is countered by the release of antiinflammatory cytokines. This anti-inflammatory response is not limited to cytokine proteins alone (such as IL-10) but also includes the release of soluble cytokine receptors (sTNFR-1 and -2) and cytokine receptor antagonists (IL-1 receptor antagonist) [3]. This complex balance between pro- and antiinflammatory molecules is likely to influence outcome following many pathophysiological insults, including cardiac surgery.

While in some respects the coagulation cascade and the inflammatory response are separate processes, they are closely interconnected in acute disease. The activation of coagulation is a key component of the acute inflammatory response and vice versa, with the endothelium intricately involved in both processes. Pro-inflammatory cytokines are released at sites of local inflammation, leading to activation of the endothelium and initiation of the coagulation cascade.

Cardiac surgery (in particular, cardiopulmonary bypass) induces activation of the immune system by factors that include (but are not limited to) contact activation of immunological cells, ischaemia-reperfusion injury, and endotoxaemia.

$\mathrm{IL}=$ interleukin; SIRS = systemic inflammatory response syndrome; sTNFR-1 = soluble tumour necrosis factor- 1 ; TNF $=$ tumour necrosis factor. 
Once activated, the immune system amplifies its response with activation of complement, cytokine production, coagulation/fibrinolysis, endothelium, and the cellular immune system. All of these processes, if unchecked, may lead to the development of SIRS and a poorer outcome in this patient group.

The use of tranexamic acid in the study by Jimenez and colleagues [1] to attenuate inflammation reveals a glimpse of how the fibrinolytic and pro-inflammatory responses are interlinked. The interplay of other major pro- and antiinflammatory mediators is worthy of further study in this patient group (in particular, TNF and IL-10) to further elucidate the pathways involved and highlight the biological targets involved in this attenuation of the inflammatory response. Their study also, perhaps more importantly, yields a practical, clinically applicable method of influencing these often catastrophic cascades that, once initiated, can lead to significant mortality and morbidity in subgroups of patients following cardiac surgery.

In the last two decades, much scientific effort has led us closer to a definition of the molecular basis of the immunological response to biological insults and to the development of specific 'targeted therapies' such as anti-TNF treatment for rheumatoid arthritis. Each additional insight into the control of inflammation and its interaction with other biological responses, such as coagulation and fibrinolysis, has the potential to lead to more effective interventions for the clinician perioperatively and in the management of critically ill patients.

\section{Competing interests}

The author declares that they have no competing interests.

\section{References}

1. Jimenez JJ, Iribarren JL, Lorente L, Rodriguez JM, Hernandez D, Nassar I, Perez R, Brouard M, Milena A, Martinez R, Mora ML: Tranexamic acid attenuates inflammatory response in cardiopulmonary bypass surgery through blockade of fibrinolysis: a case control study followed by a randomized double-blind controlled trial. Crit Care 2007, 11:R117.

2. Feldmann M, Steinman L: Design of effective immunotherapy for human autoimmunity. Nature 2005, 435:612-619.

3. Meduri GU, Headley S, Kohler G, Stentz F, Tolley E, Umberger R, Leeper K: Persistent elevation of inflammatory cytokines predicts a poor outcome in ARDS. Chest 1995, 107:1062-1073.

4. Kawamura T, Wakausawa R, Okada K, Inada S: Elevation of cytokines during open heart surgery with cardiopulmonary bypass: participation of interleukin 8 and 6 in reperfusion injury. Can J Anaesth 1993, 40:1016-1021.

5. Laffey JG, Boylan JF, Cheng DC: The systemic inflammatory response to cardiac surgery: implications for the anesthesiologist. Anesthesiology 2002, 97:215-252. 